\title{
Dissipativity verification with guarantees for polynomial systems from noisy input-state data
}

\author{
Tim Martin and Frank Allgöwer*
}

\begin{abstract}
In this paper, we investigate the verification of dissipativity properties for polynomial systems without an explicitly identified model but directly from noise-corrupted measurements. Contrary to most data-driven approaches for nonlinear systems, we determine dissipativity properties over all finite time horizons using noisy input-state data. To this end, we propose two noise characterizations to deduce two data-based set-membership representations of the ground-truth system. Each representation then serves as a framework to derive computationally tractable conditions to verify dissipativity properties with rigorous guarantees from noise-corrupted data using sum of squares (SOS) optimization.
\end{abstract}

\section{INTRODUCTION}

$\mathbf{T}$ HE standard approach to obtain a controller for nonlinear systems requires to retrieve a sufficiently precise model and the application of nonlinear controller design techniques [1]. However, the identification of nonlinear systems is in general time consuming and requires often expert knowledge. Hence, the interest on data-driven controller design techniques, where the controller is deduced without identifying a model but directly from measured data of the system, has risen recently.

One well-elaborated theory for the controller design of nonlinear systems are dissipativity properties [2] which give rise to stabilizing control laws as the small gain theorem [1] (Theorem 5.6). Since these system properties give insight to the system and facilitate a controller design without knowledge of the system, the verification of these properties from measured trajectories can be leveraged to a data-driven controller design with stability and performance guarantees.

For linear time-invariant (LTI) systems, [3] determines dissipativity properties over a data-depended finite time horizon from a noise-free single input-output trajectory. By exploiting the set-membership representation of an unknown LTI system by noisy input-state samples from [4], [5] provides guaranteed dissipativity properties over all finite time horizons as defined in [2] and required for, e.g., the small gain theorem. For nonlinear systems, [6] is tailored to estimate certain dissipativity properties over a data-depended finite time horizon, as the $\mathcal{L}_{2}$ gain or conic relations [7], from a large number of input-output trajectories based on the Lipschitz constant of the system operator. To reduce the amount of required data, [8] proposes sequential experiments to improve iteratively the accuracy of a non-parametric data-based Lipschitz approximation of the

*T. Martin and F. Allgöwer are with the Institute for Systems Theory and Automatic Control, University of Stuttgart. This work was funded by Deutsche Forschungsgemeinschaft (DFG, German Research Foundation) under Germany's Excellence Strategy - EXC 2075 - 390740016. For correspondence, mailto: tim.martin@ist.uni-stuttgart.de. system operator. Nevertheless, the amount of data might be still too large for a real application as also indicated by bounds on the sampling complexity from [9].

For that reason, we establish in this paper a data-based framework, which is based on a set-membership approach for polynomial systems and is more data-efficient than [6] and [8], to determine dissipativity properties over all finite time horizons. Contrary to [10], we consider polynomial systems in discrete time and measurements in presence of noise. By characterizing this noise by two distinct descriptions, we propose two data-based set-membership representations of the ground-truth system which constitute two frameworks to deduce computationally tractable conditions for verifying dissipativity properties using sum of squares (SOS) optimization. The first noise description bounds the noise signal in each time step which is commonly assumed, e.g., in setmembership identification [11]. This characterization yields for the verification of dissipativity properties with polynomial supply rates an SOS optimization problem which can be solved by semi-definite programming using standard SOS techniques [12]. Since the complexity of this SOS optimization problem increases for additional samples, the second ansatz characterizes the noise by a single cumulative property. This approach was first introduced in [4] and yields a feasibility condition of a linear matrix inequality (LMI) to verify $(Q, S, R)$-dissipativity.

\section{PRELIMINARIES}

In this section, we introduce the notion of SOS polynomials and matrices and formulate the problem of verifying dissipativity properties for unidentified polynomial systems from noise-corrupted input-state data.

\section{A. SOS optimization}

For a vectorial index $\alpha=\left[\begin{array}{lll}\alpha_{1} & \cdots & \alpha_{n}\end{array}\right]^{T} \in \mathbb{N}_{0}^{n}$ and a vector $x=\left[\begin{array}{lll}x_{1} & \cdots & x_{n}\end{array}\right]^{T} \in \mathbb{R}^{n}$, we write $|\alpha|=\alpha_{1}+\cdots+$ $\alpha_{n}$, the monomial $x^{\alpha}=x_{1}^{\alpha_{1}} \cdots x_{n}^{\alpha_{n}}$, and $\mathbb{R}[x]$ for the set of all polynomials $p$ in $x$, i.e.,

$$
p(x)=\sum_{\alpha \in \mathbb{N}_{0}^{n},|\alpha| \leq d} a_{\alpha} x^{\alpha},
$$

with real coefficients $a_{\alpha} \in \mathbb{R} . d \in \mathbb{N}_{0}$ corresponds to the degree of the polynomial if there is an $a_{\alpha} \neq 0$ with $|\alpha|=d$. Furthermore, we denote $\mathbb{R}[x]^{m}$ as the set of all $m$-dimensional vectors with entries in $\mathbb{R}[x]$ and $\mathbb{R}[x]^{r \times s}$ as the set of all $r \times s$ matrices with entries in $\mathbb{R}[x]$. The degree of a polynomial matrix is the largest degree of its elements. 
Definition 1 (SOS matrix). A polynomial matrix $P \in \mathbb{R}[x]^{r \times r}$ with even degree is called an SOS matrix if there exists a matrix $Q \in \mathbb{R}[x]^{s \times r}$ such that $P=Q^{T} Q$. Moreover, let the set of all $r \times r$-SOS matrices be denoted by $\operatorname{SOS}[x]^{r \times r}$. For $r=1, P$ is called SOS polynomial.

SOS matrices are computationally attractive as we can verify whether a polynomial matrix is an SOS matrix by an LMI feasibility condition which deduces from the following square matricial representation [13].

Proposition 2. A polynomial matrix $P \in \mathbb{R}[x]^{r \times r}$ is an SOS matrix if and only if there exist a real matrix $X \succeq 0$ and a vector $Z \in \mathbb{R}[x]^{\beta}$ containing monomials of $x$ such that

$$
P=\left[Z \otimes I_{r}\right]^{T} X\left[Z \otimes I_{r}\right],
$$

where $I_{r}$ denotes the $r \times r$-identity matrix and $\otimes$ corresponds to the Kronecker product.

Proof. The statement follows from the Gram matrix method [14]. A detailed proof can be found in [13].

In our application of SOS optimization, we are confronted to verify that a polynomial $p \in \mathbb{R}[x]$ is non-negative for all $x \in\left\{x \in \mathbb{R}^{n}: c_{1}(x) \geq 0, \ldots, c_{k}(x) \geq 0\right\}$ with $c_{i} \in \mathbb{R}[x]$. We can boil down this problem to an LMI feasibility condition using the following SOS relaxation from [15].

Proposition 3 (SOS relaxation). A polynomial $p \in \mathbb{R}[x]$ is non-negative for all $x \in\left\{x \in \mathbb{R}^{n}: c_{1}(x) \geq 0, \ldots, c_{k}(x) \geq\right.$ $0\}$ with $c_{i} \in \mathbb{R}[x]$ if there exist SOS polynomials $t_{i} \in$ $\operatorname{SOS}[x], i=1, \ldots, k$ such that $p-\sum_{i=1}^{k} t_{i} c_{i} \in \operatorname{SOS}[x]$.

Proof. A proof based on the Positivstellensatz can be found in [15] (Lemma 2.1).

\section{B. Problem setup}

We consider the nonlinear discrete-time system with polynomial dynamics

$$
x(t+1)=f(x(t), u(t)), f \in \mathbb{R}[x, u]^{n}
$$

and state-input constraints $(x, u) \in \mathbb{P}$ with non-empty set

$$
\begin{array}{r}
\mathbb{P}=\left\{(x, u) \in \mathbb{R}^{n} \times \mathbb{R}^{m}: p_{i}(x, u) \leq 0,\right. \\
p_{i} \in \mathbb{R}[x, u], \\
i=1, \ldots, c\} .
\end{array}
$$

The goal of this paper is the derivation of computationally tractable conditions to check whether system (1) is dissipative on (2) without identifying a model but directly from inputstate data. Since dissipativity properties are originally defined for continuous-time unconstrained systems in [2], we specify a suitable notion of dissipativity for discrete-time systems under constraints.

Definition 4 (Dissipativity). System (1) is dissipative on $\mathbb{P} \subseteq$ $\mathbb{R}^{n} \times \mathbb{R}^{m}$ with respect to the given supply rate $s: \mathbb{P} \rightarrow \mathbb{R}$ if there exists a continuous storage function $\lambda: \mathbb{X} \rightarrow \mathbb{R}_{\geq 0}$ such that

$$
\lambda(f(x, u))-\lambda(x) \leq s(x, u), \quad \forall(x, u) \in \mathbb{P}
$$

where $\mathbb{X} \subseteq \mathbb{R}^{n}$ denotes the projection of $\mathbb{P}$ on the state-space $\mathbb{R}^{n}$. Moreover, the system is called $(Q, S, R)$-dissipative if it is dissipative with respect to the supply rate

$$
s(x, u)=\left[\begin{array}{l}
x \\
u
\end{array}\right]^{T}\left[\begin{array}{cc}
Q & S \\
S^{T} & R
\end{array}\right]\left[\begin{array}{l}
x \\
u
\end{array}\right]
$$

While the verification of dissipativity inequality (3) for a (known) polynomial system (1) and polynomial supply rate using SOS optimization is well-investigated [12], dissipativity verification of an unidentified polynomial system directly from noisy data as formulated next hasn't been analyzed yet.

Suppose that an upper bound on the degree of $f$ is known while its coefficients are unidentified. Then the system dynamics (1) can be represented by

$$
f(x, u)=A z(x, u)=\left(I_{n} \otimes z(x, u)^{T}\right) a,
$$

where $z \in \mathbb{R}[x, u]^{\ell}$ contains at least all monomials of $f$ according to the known upper bound on the degree of $f$. $A \in \mathbb{R}^{n \times \ell}$ and $a=\operatorname{vec}\left(A^{T}\right) \in \mathbb{R}^{n \ell}$, where vec denotes the vectorization of a matrix by stacking its columns, contain the unknown coefficients. Furthermore, we assume the access to noisy input-state data

$$
\left\{\left(\tilde{x}_{i}^{+}, \tilde{x}_{i}, \tilde{u}_{i}\right)_{i=1, \ldots, D}\right\}
$$

satisfying $\tilde{x}_{i}^{+}=f\left(\tilde{x}_{i}, \tilde{u}_{i}\right)+\tilde{d}_{i}$. Note that we measure the state $\tilde{x}_{i}^{+}$from the underlying system, i.e., $\tilde{x}_{i}^{+}=x_{i}^{+}+d_{i}$ with $x_{i}^{+}=f\left(x_{i}, u_{i}\right)$, the true state $x_{i} \neq \tilde{x}_{i}$, and input $u_{i} \neq \tilde{u}_{i}$ and where $d_{i} \in \mathbb{R}^{n}$ summarizes the uncertainty due to measurement noise. Therefore, $\tilde{d}_{i}=d_{i}+f\left(x_{i}, u_{i}\right)-f\left(\tilde{x}_{i}, \tilde{u}_{i}\right)$, i.e., the noise vector $\tilde{d}_{i}$ contains the effect of $d_{i}$ and analogously to [11] the difference when applying the dynamics to the uncertain state $\tilde{x}_{i}$ and input $\tilde{u}_{i}$ instead of the true state $x_{i}$ and input $u_{i}$. As clarified in [5], we could also study noise $\tilde{d}_{i}$ that affects through a matrix $B$ to include addition knowledge on its influence.

In the sequel, we characterize the noise $\tilde{d}_{i}, i=1, \ldots, D$ more precisely to derive data-based set-membership representations of the unidentified polynomial system (1).

\section{DATA-DRIVEN DISSIPATIVITY VERIFICATION FOR SEPARATELY BOUNDED NOISE}

In this section, we develop a framework for dissipativity verification of polynomial system (1) from noise-corrupted data (5) if the noise is bounded explicitly in each time step as specified in the following assumption.

Assumption 5 (Separately bounded noise). For the measured data (5), suppose that for $i=1, \ldots, D$

$$
\tilde{d}_{i} \in \mathcal{D}_{i}^{\mathrm{SB}}=\left\{d \in \mathbb{R}^{n}: \delta_{i}(d) \leq 0, \delta_{i} \in \mathbb{R}[d]\right\},
$$

where $\mathcal{D}_{i}^{\mathrm{SB}}$ is bounded.

The noise characterization in Assumption 5 seems to be general and incorporates, e.g., quadratically bounded noise

$$
\delta_{i}(d)=\left[\begin{array}{l}
d \\
1
\end{array}\right]^{T}\left[\begin{array}{ll}
\Delta_{1} & \Delta_{2} \\
\Delta_{2}^{T} & \Delta_{3}
\end{array}\right]\left[\begin{array}{l}
d \\
1
\end{array}\right], \Delta_{1} \succ 0, \Delta_{3} \leq 0 .
$$


Moreover, Assumption 5 includes noise with bounded amplitude $\delta_{i}(d)=d^{T} d-\epsilon^{2}$ and noise that exhibits a fixed signalto-noise-ratio $\delta_{i}(d)=d^{T} d-\tilde{\epsilon}^{2} \tilde{x}_{i}^{T} \tilde{x}_{i}$ which are frequently assumed in system identification [11].

To derive a data-based set-membership representation of the ground-truth system (1) which is the basis to verify dissipativity properties without identifying an explicit model, we next define the set of all systems parametrized by $a$

$$
x(t+1)=\underbrace{\left(I_{n} \otimes z(x, u)^{T}\right)}_{=: Z(x, u)} a,
$$

with unidentified coefficient vector $a \in \mathbb{R}^{n \ell}$ and known vector $z \in \mathbb{R}[x, u]^{\ell}$, which explain the data (5).

Definition 6 (Feasible system set). The set of all systems (8) admissible with the measured data (5) for separately bounded noise (6) is given by the feasible system set $\operatorname{FSS}_{\mathrm{SB}}=\{Z a \in$ $\left.\mathbb{R}[x, u]^{n}: a \in \Sigma_{\mathrm{SB}}\right\}$ with $\Sigma_{\mathrm{SB}}=\left\{a \in \mathbb{R}^{n \ell}: \forall i \in\right.$ $\{1, \ldots, D\} \quad \exists \tilde{d}_{i} \in \mathcal{D}_{i}^{\mathrm{SB}}$ satisfying $\left.\tilde{x}_{i}^{+}=Z\left(\tilde{x}_{i}, \tilde{u}_{i}\right) a+\tilde{d}_{i}\right\}$.

Since the samples (5) satisfy $\tilde{x}_{i}^{+}=f\left(\tilde{x}_{i}, \tilde{u}_{i}\right)+\tilde{d}_{i}$ with $\tilde{d}_{i} \in \mathcal{D}_{i}^{\mathrm{SB}}$ by assumption, the ground-truth system is an element of $\mathrm{FSS}_{\mathrm{SB}}$, i.e., $f \in \mathrm{FSS}_{\mathrm{SB}}$. Thereby, $\mathrm{FSS}_{\mathrm{SB}}$ is a set-membership representation of the ground-truth system (1). Analogously to [4], we deduce in the following lemma a databased description of $\mathrm{FSS}_{\mathrm{SB}}$.

Lemma 7. The set of all coefficients $\Sigma_{\mathrm{SB}}$ for which system (8) explains the measured data set (5) for separately bounded noise (6) is equivalent to

$$
\left\{a \in \mathbb{R}^{n \ell}: \delta_{i}\left(\tilde{x}_{i}^{+}-Z\left(\tilde{x}_{i}, \tilde{u}_{i}\right) a\right) \leq 0, i=1, \ldots, D\right\}
$$

with the data-dependent polynomials $\delta_{i}\left(\tilde{x}_{i}^{+}-Z\left(\tilde{x}_{i}, \tilde{u}_{i}\right) a\right) \in$ $\mathbb{R}[a], i=1, \ldots, D$.

Proof. If $a \in \Sigma_{\mathrm{SB}}$ then there exist realizations of the noise $\tilde{d}_{i}, i=1, \ldots, D$ such that $\tilde{x}_{i}^{+}=Z\left(\tilde{x}_{i}, \tilde{u}_{i}\right) a+\tilde{d}_{i}$ and $\delta_{i}\left(\tilde{d}_{i}\right) \leq$ 0 . Combining both yields (9).

To prove the converse, suppose that $a$ is an element of (9). Then construct $\tilde{d}_{i}, i=1, \ldots, D$ such that $\tilde{x}_{i}^{+}=Z\left(\tilde{x}_{i}, \tilde{u}_{i}\right) a+$ $\tilde{d}_{i}$. Since $a$ satisfies (9), $\tilde{d}_{i}, i=1, \ldots, D$ satisfy (6), and hence $a \in \Sigma_{\mathrm{SB}}$.

Since FSS $_{\mathrm{SB}}$ contains the ground-truth systems, (1) is dissipative if all systems of the feasible system set FSS $_{\mathrm{SB}}$ are dissipative. Based on this idea, the following theorem provides a data-based SOS condition for the verification of dissipativity properties without an identified model of (1).

Theorem 8. Let the data samples (5) satisfy Assumption 5 Then system (1) is dissipative on (2) w.r.t. the given supply rate $s \in \mathbb{R}[x, u]$ if there exist a storage function $\lambda \in \operatorname{SOS}[x]$ and polynomials $s_{i} \in \operatorname{SOS}[x, u, a], i=1, \ldots, c$ and $t_{i} \in$ $\operatorname{SOS}[x, u, a], i=1, \ldots, D$ such that $\psi \in \operatorname{SOS}[x, u, a]$ with

$$
\begin{aligned}
& \psi(x, u, a)=s(x, u)-\lambda(Z(x, u) a)+\lambda(x)+\ldots \\
& \sum_{i=1}^{D} \delta_{i}\left(\tilde{x}_{i}^{+}-Z\left(\tilde{x}_{i}, \tilde{u}_{i}\right) a\right) t_{i}(x, u, a)+\sum_{i=1}^{c} p_{i}(x, u) s_{i}(x, u, a) .
\end{aligned}
$$

Proof. By Definition 4, all systems of the feasible system set $\mathrm{FSS}_{\mathrm{SB}}$, and hence system (1), are dissipative on (2) if there exists a continuous storage function $\lambda: \mathbb{X} \rightarrow \mathbb{R}_{\geq 0}$ such that

$$
s(x, u)-\lambda(Z(x, u) a)+\lambda(x) \geq 0
$$

for all $(x, u) \in \mathbb{P}$ and all $a \in \Sigma_{\mathrm{SB}}$. Since the sets $\mathbb{P}$ and $\Sigma_{\mathrm{SB}}$ are defined by polynomial inequalities in (2) and Lemma 7 respectively, we can apply Proposition 3 to conclude that (10) holds if there exist a storage function $\lambda \in \operatorname{SOS}[x]$ and SOS polynomials $s_{i} \in \operatorname{SOS}[x, u, a], i=1, \ldots, c$ and $t_{i} \in$ $\operatorname{SOS}[x, u, a], i=1, \ldots, D$ such that $\psi \in \operatorname{SOS}[x, u, a]$.

Even though $Z(x, u) a$ is an unidentified polynomial vector in $\mathbb{R}[x, u]^{n}$, it is a known polynomial vector in $\mathbb{R}[x, u, a]^{n}$. For that reason, we can verify $\psi \in \operatorname{SOS}[x, u, a]$ as an SOS problem with free variables $x, u$, and $a$ by applying standard SOS solvers, e.g., [16]. For quadratically bounded noise (7), we can achieve an SOS condition independent of $a$.

Corollary 9. Let the data samples (5) satisfy Assumption 5 with $\delta_{i}$ from (7). Then system (11) is dissipative on (2) with respect to the supply rate $s \in \mathbb{R}[x, u]$ if there exist a storage function $\lambda(x)=x^{T} P x, P \succeq 0$ and polynomials $t_{i} \in \operatorname{SOS}[x, u], i=1, \ldots, D$ and $s_{i}(x, u, a)=$ $\left[\begin{array}{ll}a^{T} & 1\end{array}\right] S_{i}(x, u)\left[\begin{array}{ll}a^{T} & 1\end{array}\right]^{T}, i=1, \ldots, c$, with $S_{i} \in$ $\operatorname{SOS}[x, u]^{(n \ell+1) \times(n \ell+1)} \operatorname{such}$ that $\Psi \in \operatorname{SOS}[x, u]^{(n \ell+1) \times(n \ell+1)}$ with

$$
\begin{aligned}
\Psi(x, u) & =\sum_{i=1}^{c} p_{i}(x, u) S_{i}(x, u)+\sum_{i=1}^{D} Q_{i} t_{i}(x, u) \\
& +\left[\begin{array}{cc}
-Z(x, u)^{T} P Z(x, u) & 0 \\
0 & s(x, u)+x^{T} P x
\end{array}\right]
\end{aligned}
$$

and the data-dependent matrices

$$
Q_{i}=\left[\begin{array}{cc}
\tilde{Z}_{i}^{T} \Delta_{1} \tilde{Z}_{i} & -\tilde{Z}_{i}^{T}\left(\Delta_{1} \tilde{x}_{i}^{+}+\Delta_{2}\right) \\
-\left(\tilde{x}_{i}^{+{ }^{T}} \Delta_{1}+\Delta_{2}^{T}\right) \tilde{Z}_{i} & {\left[\begin{array}{c}
\tilde{x}_{i}^{+} \\
1
\end{array}\right]^{T}\left[\begin{array}{ll}
\Delta_{1} & \Delta_{2} \\
\Delta_{2}^{T} & \Delta_{3}
\end{array}\right]\left[\begin{array}{c}
\tilde{x}_{i}^{+} \\
1
\end{array}\right]}
\end{array}\right]
$$

using the abbreviation $Z\left(\tilde{x}_{i}, \tilde{u}_{i}\right)=\tilde{Z}_{i}$.

Proof. Note that the quadratically bounded noise (7) yields $\delta_{i}\left(\tilde{x}_{i}^{+}-Z\left(\tilde{x}_{i}, \tilde{u}_{i}\right) a\right)=\left[\begin{array}{ll}a^{T} & 1\end{array}\right] Q_{i}\left[\begin{array}{ll}a^{T} & 1\end{array}\right]^{T}$. Then pursuing the proof of Theorem [8 system (11) is dissipative if there exist a $P \succeq 0, t_{i} \in \operatorname{SOS}[x, u], i=1, \ldots, D$, and $S_{i} \in$ $\operatorname{SOS}[x, u]^{(n \ell+1) \times(n \ell+1)}, i=1, \ldots, c$ such that

$$
\left[\begin{array}{l}
a \\
1
\end{array}\right]^{T} \Psi(x, u)\left[\begin{array}{l}
a \\
1
\end{array}\right] \in \operatorname{SOS}[x, u, a] .
$$

If $\Psi \in \operatorname{SOS}[x, u]^{(n \ell+1) \times(n \ell+1)}$ then there exists a $\Phi \in$ $\mathbb{R}[x, u]^{q \times(n \ell+1)}$ with $\Psi=\Phi^{T} \Phi$ by Definition 1 Therefore, (11) is an SOS polynomial by Definition 1.

For a closer look on Theorem 8 and Corollary 9 we refer to Section $\mathrm{V}$ and finish this section with an extension of Theorem 8 and Corollary 9 , respectively.

Remark 10. To exclude time-varying coefficients $a(t) \in \Sigma_{\mathrm{SB}}$ in Theorem 8 and Corollary 9 and hence to reduce their conservatism, we could consider parametrized storage functions $\lambda \in \operatorname{SOS}[x, a]$ and the dissipativity inequality

$$
\lambda(Z(x, u) a, a)-\lambda(x, a) \leq s(x, u), \forall(x, u) \in \mathbb{P}, \forall a \in \Sigma_{\mathrm{SB}} .
$$




\section{DATA-DRIVEN DISSIPATIVITY VERIFICATION FOR CUMULATIVELY BOUNDED NOISE}

We again tackle the problem of verifying whether the unidentified polynomial system (1) is dissipative by means of noisy data. However, instead of bounding the noise separately in time as in the previous section, the noise is characterized by one property that bounds cumulatively the noise realizations of the data samples (5), which was first proposed in [4].

Assumption 11 (Cumulatively bounded noise). For the measured data (5), suppose that the matrix $\tilde{D}=\left[\begin{array}{lll}\tilde{d}_{1} & \cdots & \tilde{d}_{D}\end{array}\right]$ is an element of

$$
\mathcal{D}^{\mathrm{CB}}=\left\{F \in \mathbb{R}^{n \times D}:\left[\begin{array}{c}
F^{T} \\
I_{n}
\end{array}\right]^{T}\left[\begin{array}{cc}
\Delta_{1} & \Delta_{2} \\
\Delta_{2}^{T} & \Delta_{3}
\end{array}\right]\left[\begin{array}{c}
F^{T} \\
I_{n}
\end{array}\right] \prec 0\right\}
$$

with $\Delta_{1} \succeq 0$.

By Assumption 11, all noise realizations $\tilde{d}_{1}, \ldots, \tilde{d}_{D}$ are cumulatively bounded as $\Delta_{1} \succeq 0$. Exemplary, (12) incorporates noise with (strictly) bounded energy $\sum_{i=1}^{D} \tilde{d}_{i}^{T} \tilde{d}_{i}<\delta_{\mathrm{e}}^{2}$ by $\tilde{D} \tilde{D}^{T} \prec \delta_{\mathrm{e}}^{2} I_{n}$.

Analogously to [4] and Section [I-A] combining Assumption 11, data samples (5), and the system dynamics

$$
x(t+1)=A z(x(t), u(t)),
$$

with unidentified coefficients $A \in \mathbb{R}^{n \times \ell}$, yields a data-based set-membership representation of the ground-truth system (1) which is summarized in the following definition and lemma.

Definition 12 (Feasible system set). The set of all systems (13) admissible with the measured data set (5) for cumulatively bounded noise (12) is given by the feasible system set $\mathrm{FSS}_{\mathrm{CB}}=\left\{A z \in \mathbb{R}[x, u]^{n}: A \in \Sigma_{\mathrm{CB}}\right\}$ with $\Sigma_{\mathrm{CB}}=$ $\left\{A \in \mathbb{R}^{n \times \ell}: \exists\left[\begin{array}{lll}\tilde{d}_{1} & \cdots & \tilde{d}_{D}\end{array}\right] \in \mathcal{D}^{\mathrm{CB}}\right.$ satisfying $\tilde{x}_{i}^{+}=$ $\left.A z\left(\tilde{x}_{i}, \tilde{u}_{i}\right)+\tilde{d}_{i}, i=1, \ldots, D\right\}$.

Lemma 13. The set of all coefficients $\Sigma_{\mathrm{CB}}$ for which system (13) explains the measured data set (5) for cumulatively bounded noise (12) is equivalent to

$$
\left\{A \in \mathbb{R}^{n \times \ell}:\left[\begin{array}{c}
A^{T} \\
I_{n}
\end{array}\right]^{T}\left[\begin{array}{cc}
\tilde{\Delta}_{1} & \tilde{\Delta}_{2} \\
\tilde{\Delta}_{2}^{T} & \tilde{\Delta}_{3}
\end{array}\right]\left[\begin{array}{c}
A^{T} \\
I_{n}
\end{array}\right] \prec 0\right\}
$$

with the data-dependent matrices $\tilde{X}^{+}=\left[\begin{array}{lll}\tilde{x}_{1}^{+} & \cdots & \tilde{x}_{D}^{+}\end{array}\right]$, $\tilde{Z}=\left[\begin{array}{lll}z\left(\tilde{x}_{1}, \tilde{u}_{1}\right) & \cdots & z\left(\tilde{x}_{D}, \tilde{u}_{D}\right)\end{array}\right]$, and

$$
\begin{aligned}
& {\left[\begin{array}{cc}
\tilde{\Delta}_{1} & \tilde{\Delta}_{2} \\
\tilde{\Delta}_{2}^{T} & \tilde{\Delta}_{3}
\end{array}\right]} \\
& =\left[\begin{array}{cc}
\tilde{Z} \Delta_{1} \tilde{Z}^{T} & -\tilde{Z}\left(\Delta_{1} \tilde{X}^{+{ }^{T}}+\Delta_{2}\right) \\
-\left(\tilde{X}^{+} \Delta_{1}+\Delta_{2}^{T}\right) \tilde{Z}^{T} & {\left[\begin{array}{c}
\tilde{X}^{+{ }^{T}} \\
I_{n}
\end{array}\right]^{T}\left[\begin{array}{cc}
\Delta_{1} & \Delta_{2} \\
\Delta_{2}^{T} & \Delta_{3}
\end{array}\right]\left[\begin{array}{c}
\tilde{X}^{+{ }^{T}} \\
I_{n}
\end{array}\right]}
\end{array}\right] .
\end{aligned}
$$

Proof. The statement follows analogously to [4] (Lemma 4) and the proof of Lemma 7 respectively.

Since the data-based description of $\Sigma_{\mathrm{CB}}$ in Lemma 13 provides a bound on $A^{T}$ instead of $A$ as will be required for the verification of the "primal" dissipativity inequality (3), we introduce the dual version of (14) as in [5].
Lemma 14. Suppose that Assumption 11 holds and the inverse

$$
\left[\begin{array}{cc}
-\tilde{\Delta}_{1} & \tilde{\Delta}_{2} \\
\tilde{\Delta}_{2}^{T} & -\tilde{\Delta}_{3}
\end{array}\right]^{-1}=:\left[\begin{array}{cc}
\bar{\Delta}_{1} & \bar{\Delta}_{2} \\
\bar{\Delta}_{2}^{T} & \bar{\Delta}_{3}
\end{array}\right]
$$

exists. Then any matrix $A \in \mathbb{R}^{n \times \ell}$ is an element of $\Sigma_{\mathrm{CB}}$ if and only if

$$
A \in \bar{\Sigma}_{\mathrm{CB}}=\left\{A \in \mathbb{R}^{n \times \ell}:\left[\begin{array}{c}
I_{\ell} \\
A
\end{array}\right]^{T}\left[\begin{array}{cc}
\bar{\Delta}_{1} & \bar{\Delta}_{2} \\
\bar{\Delta}_{2}^{T} & \bar{\Delta}_{3}
\end{array}\right]\left[\begin{array}{c}
I_{\ell} \\
A
\end{array}\right] \prec 0\right\} .
$$

Proof. Since the samples (5) satisfy $\tilde{x}_{i}^{+}=f\left(\tilde{x}_{i}, \tilde{u}_{i}\right)+\tilde{d}_{i}$ with $\left[\begin{array}{lll}\tilde{d}_{1} & \cdots & \tilde{d}_{D}\end{array}\right] \in \mathcal{D}^{\mathrm{CB}}$ by assumption, the coefficient matrix $A_{\mathrm{gt}}$ of the ground-truth system (1), i.e., $f(x, u)=A_{\mathrm{gt}} z(x, u)$, is an element of $\Sigma_{\mathrm{CB}}$. Together with $\Delta_{1} \succeq 0$, the dualization lemma [17] implies that $\left(A_{\mathrm{gt}} \in \bar{\Sigma}_{\mathrm{CB}}\right.$ and) $\bar{\Delta}_{3} \succeq 0$. Thereby, any matrix $A \in \mathbb{R}^{n \times \ell}$ satisfies $A \in \Sigma_{\mathrm{CB}}$ if and only if $A \in$ $\bar{\Sigma}_{\mathrm{CB}}$ again by the dualization lemma.

By Lemma 14, the feasible system sets $\mathrm{FSS}_{\mathrm{CB}}$ and $\overline{\mathrm{FSS}}_{\mathrm{CB}}=$ $\left\{A z \in \mathbb{R}[x, u]^{n}: A \in \bar{\Sigma}_{\mathrm{CB}}\right\}$ are equivalent and contain the ground-truth system (1). Therefore, we can derive analogously to Section $\Pi$-A a condition to verify dissipativity properties of polynomial system (1) without identifying a model but directly from noisy input-state measurement.

Theorem 15. Suppose that the data samples (5) satisfy Assumption 11, the inverse (15) exists, the state-inputs constraints (2) are specified by

$$
p_{i}(x, u)=\left[\begin{array}{c}
z(x, u) \\
1
\end{array}\right]^{T} P_{i}\left[\begin{array}{c}
z(x, u) \\
1
\end{array}\right], i=1, \ldots, c,
$$

with $P_{i} \in \mathbb{R}^{(\ell+1) \times(\ell+1)}$ and, without loss of generality, there exist matrices $T_{x} \in \mathbb{R}^{n \times \ell}$ and $T \in \mathbb{R}^{(n+m) \times \ell}$ such that $x=$ $T_{x} z$ and $\left[\begin{array}{l}x \\ u\end{array}\right]=T z$. Then system (1) is $(Q, S, R)$-dissipative on (2) with quadratic constraints (16) if the LMI (17) holds for a storage function $\lambda(x)=x^{T} P x, P \succeq 0$, a constant $\tau \geq 0$, and polynomials $z_{i} \tau_{i} \in \operatorname{SOS}[x, u], i=1, \ldots, c$ with a vector of monomials $z_{i} \in \mathbb{R}[x, u]^{1 \times \beta}$, to-be-optimized coefficients $\tau_{i} \in \mathbb{R}^{\beta}$, and a linear mapping $\tilde{P}_{i}: \mathbb{R}^{\beta} \rightarrow \mathbb{R}^{(\ell+1) \times(\ell+1)}$ with

$$
z_{i} \tau_{i}\left[\begin{array}{c}
z \\
1
\end{array}\right]^{T} P_{i}\left[\begin{array}{l}
z \\
1
\end{array}\right]=\left[\begin{array}{c}
z \\
1
\end{array}\right]^{T} \tilde{P}_{i}\left(\tau_{i}\right)\left[\begin{array}{l}
z \\
1
\end{array}\right]
$$

Proof. Since the ground-truth system (1) is an element of $\mathrm{FSS}_{\mathrm{CB}}$ and $\overline{\mathrm{FSS}}_{\mathrm{CB}}$ by Lemma 14 system (1) is $(Q, S, R)$ dissipative on (2) with quadratic constraints (16) if there exists a storage function $\lambda(x)=x^{T} P x, P \succeq 0$ such that

$$
\begin{aligned}
& x^{T} P x-z(x, u)^{T} A^{T} P A z(x, u)+\left[\begin{array}{l}
x \\
u
\end{array}\right]^{T}\left[\begin{array}{ll}
Q & S \\
S^{T} & R
\end{array}\right]\left[\begin{array}{l}
x \\
u
\end{array}\right] \geq 0, \\
& \forall(x, u):\left[\begin{array}{c}
z(x, u) \\
1
\end{array}\right]^{T} P_{i}\left[\begin{array}{c}
z(x, u) \\
1
\end{array}\right] \leq 0, i=1, \ldots, c, \forall A \in \bar{\Sigma}_{\mathrm{CB}} .
\end{aligned}
$$

With $A \in \bar{\Sigma}_{\mathrm{CB}}$ implying that for all $(x, u) \in \mathbb{R}^{n} \times \mathbb{R}^{m}$

$$
z(x, u)^{T}\left[\begin{array}{c}
I_{\ell} \\
A
\end{array}\right]^{T}\left[\begin{array}{ll}
\bar{\Delta}_{1} & \bar{\Delta}_{2} \\
\bar{\Delta}_{2}^{T} & \bar{\Delta}_{3}
\end{array}\right]\left[\begin{array}{c}
I_{\ell} \\
A
\end{array}\right] z(x, u) \leq 0
$$




$$
\Theta:=\left[\begin{array}{ccc}
I_{n} & 0 & 0 \\
0 & T_{x} & 0 \\
\hline 0 & T & 0 \\
\hline 0 & I_{\ell} & 0 \\
I_{n} & 0 & 0 \\
\hline 0 & I_{\ell} & 0 \\
0 & 0 & 1
\end{array}\right]^{T}\left[\begin{array}{cc|cc|cc|cc}
-P & 0 & 0 & 0 & 0 & 0 & 0 \\
0 & P & 0 & 0 & 0 & 0 & 0 \\
\hline 0 & 0 & Q & S & 0 & 0 & 0 \\
0 & 0 & S^{T} & R & 0 & 0 & 0 \\
\hline 0 & 0 & 0 & 0 & \tau \bar{\Delta}_{1} & \tau \bar{\Delta}_{2} & 0 \\
0 & 0 & 0 & 0 & \tau \bar{\Delta}_{2}^{T} & \tau \bar{\Delta}_{3} & 0 \\
\hline 0 & 0 & 0 & 0 & 0 & 0 & \sum_{i=1}^{c} \tilde{P}_{i}\left(\tau_{i}\right)
\end{array}\right]\left[\begin{array}{ccc}
I_{n} & 0 & 0 \\
0 & T_{x} & 0 \\
\hline 0 & T & 0 \\
\hline 0 & I_{\ell} & 0 \\
I_{n} & 0 & 0 \\
\hline 0 & I_{\ell} & 0 \\
0 & 0 & 1
\end{array}\right] \succeq 0
$$

we apply Proposition 3 to conclude that the conditioned dissipativity inequality (19) holds if there exist a $P \succeq 0$, a nonnegative constants $\tau$ and polynomials $z_{i} \tau_{i} \in \operatorname{SOS}[x, u], i=$ $1, \ldots, c$ with (18) satisfying

$$
L(x, u, A)^{T} \Theta L(x, u, A) \in \operatorname{SOS}[x, u, \operatorname{vec}(A)]
$$

with $L(x, u, A)=\left[\begin{array}{lll}z(x, u)^{T} A^{T} & z(x, u)^{T} & 1\end{array}\right]^{T}$. Finally, if (17) is satisfied then there exists a matrix $\Omega$ with $\Theta=\Omega^{T} \Omega$ and thus (20) is an SOS polynomial by Definition 1

In Theorem 15, dissipativity verification boils down to an LMI feasibility problem instead of an SOS problem as in Theorem 8 because we extract in (20) all monomials in $x, u$, and $\operatorname{vec}(A)$ into $L(x, u, A)$ similar to the square matricial representation in Proposition 2 However, this computational advantage comes at the cost of additional conservatism compared to Theorem 8 as Theorem 15 considers quadratic storage functions and requires in its derivation simplified multipliers for Proposition 3, e.g., $\tau$ independent of $x$ and $u$. Note that we can generalize Theorem 15 for supply rates $s(x, u)=z(x, u)^{T} Q z(x, u)$.

In Theorem 15, we consider SOS polynomials $z_{i}(x, u) \tau_{i}, i=1, \ldots, c$ instead of non-negative constants as otherwise LMI (17) becomes indefinite if $P_{i}$ contains a negative right lower element which is mostly the case, e.g., $x^{T} x \leq 1$. Note that a linear mapping $\tilde{P}_{i}$ exists as the left hand side of the quadratic decomposition (18) is linear in $\tau_{i}$. However, $\tilde{P}_{i}$ is not unique but is spanned by a linear subspace which provides additional degrees of freedom to deteriorate the conservatism of condition (17).

We conclude this section by demonstrating the flexibility of this framework by employing prior system knowledge.

Remark 16. We can take prior knowledge of the system dynamics into account by considering

$$
x(t+1)=A z_{1}(x(t), u(t))+\left[\begin{array}{ll}
\bar{A}_{1} & \bar{A}_{2}
\end{array}\right]\left[\begin{array}{l}
z_{1}(x(t), u(t)) \\
z_{2}(x(t), u(t))
\end{array}\right]
$$

with unidentified matrix $A$ and known matrices $\bar{A}_{1}$ and $\bar{A}_{2}$. The additional vector of monomials $z_{2}(x, u)$ is beneficial if, for instance, $g(x)$ of a (polynomial) control-affine system $x(t+1)=f(x(t))+g(x(t)) u(t)$ is known from some insight to the system. Moreover, $z_{2}(x, u)$ might be necessary for the quadratic decomposition (18). Note that incorporating prior knowledge as described here is also conceivable for the framework of separately bounded noise.

\section{COMPARISON OF BOTH FRAMEWORKS FOR SEPARATELY BOUNDED NOISE}

Motivated by the frequently assumed separately bounded noise $\left\|\tilde{d}_{i}\right\|_{2} \leq \epsilon_{i}$ as non-probabilistic noise description, e.g., in system identification [11], we compare in this section both previously proposed frameworks for data-driven dissipativity verification for this noise characterization.

According to [4], the cumulatively bounded noise description (12) can incorporated this separately bounded noise by $\tilde{D} \tilde{D}^{T} \preceq \sum_{i=1}^{D} \epsilon_{i}^{2} I_{n}$. However, this characterisation also facilitates, e.g., noise with bounded energy $\sum_{i=1}^{D} \tilde{d}_{i}^{T} \tilde{d}_{i} \leq \sum_{i=1}^{D} \epsilon_{i}^{2}$ which includes more noise realizations than $\left\|\tilde{d}_{i}\right\|_{2} \leq \epsilon_{i}$. Hence, Assumption 5 provides a more accurate description than Assumption 11 for the separately bounded noise $\left\|\tilde{d}_{i}\right\|_{2} \leq$ $\epsilon_{i}$, and therefore leads to a tighter set-membership representation of the ground-truth system (1). For that reason, Theorem 8 provides a less conservative condition for dissipativity verification than Theorem 15 which is indeed observed in Section VI.

Furthermore, the feasible system set FSS $_{\mathrm{SB}}$ cannot increase by considering additional data samples. Contrary, we show in Subsection VI-A that adding samples with high signal-tonoise-ratio to an original data set of $\mathrm{FSS}_{\mathrm{CB}}$ might decrease its accuracy for dissipativity verification, and hence might render LMI (17) infeasible. One explanation is that we cumulate all data samples equally weighted in (14) into one condition which corresponds to restrict all SOS polynomial multipliers $t_{i}(x, u, a), i=1, \ldots, D$ in Theorem 8 to be equal. Therefore, data samples with large noise increase the uncertainty of FSS $_{\mathrm{SB}}$. To circumvent this problem in Theorem 15, we could consider the intersection of $\mathrm{FSS}_{\mathrm{CB}}$ for the original data set and for the data set with additional data by an S-procedure argument.

Further advantages of Theorem 8 are that its accuracy can be improved by parametrized storage functions as shown in Remark 10 and general polynomial state-input constraints and supply rates can be handled.

On the other hand, the framework of cumulatively bounded noise is computationally more attractive. The verification condition in Theorem 15 boils down to an LMI condition and its complexity doesn't increase with the amount of samples as all data samples (5) are cumulated into one condition. Contrary, Theorem 8 requires one additional SOS polynomial multiplier for each sample which might yield to a non-tractable optimization problem. This issue could be circumvented by the relaxation $t_{1}(x, u, a)=\cdots=t_{D}(x, u, a)$ which then leads to a cumulative noise characterization. 
Furthermore, in our testing in Section VI system description (13) is computationally more efficient than (8) when tackling systems (4) with a large number of unidentified coefficients.

To summarize this discussion, while the framework of separately bounded noise provides a data-efficient approach for the often used bounded noise $\left\|\tilde{d}_{i}\right\|_{2} \leq \epsilon_{i}$, the framework of cumulatively bounded noise is computationally more attractive. For that reason, the latter framework should always be considered if the noise is characterized by some cumulative property.

\section{NumERICAL EXAMPLES}

To measure the conservatism of both frameworks for separately bounded noise, we apply Corollary 9 and Theorem 15 on two systems to find a guaranteed upper bound on their $\ell_{2}$-gain $\gamma$ which corresponds to the supply rate $s(x, u)=$ $\gamma^{2} u^{T} u-x^{T} x$. To this end, the SOS problem of Corollary 9 and the LMI feasibility problem of Theorem 15 are extended by the minimization over $\gamma$.

\section{A. Example 1}

We determine an upper bound on the $\ell_{2}$-gain of the polynomial system

$$
x(t+1)=-0.8 x(t)+0.1 x(t)^{2}+u(t)
$$

with state constraint $x^{2}-1 \leq 0$ and input constraint $u^{2}-0.01 \leq 0$. We receive the upper bound $\gamma \leq 10.0$ by SOS optimization exploiting the system dynamics.

To apply our data-driven methods, we draw samples (5) from a single trajectory with initial condition $x(0)=1$, input $u(t)=0.1, t \geq 0$, and a random sampled and (separately) bounded noise $\left|\tilde{d}_{i}\right| \leq 0.02$.

Considering the first three noisy data samples of the trajectory, we receive the upper bounds for the $\ell_{2}$-gain $\gamma_{\mathrm{SB}}=16.3$ from Corollary 9 and $\gamma_{\mathrm{CB}}=17.1$ from Theorem 15

As stated in Section D additional data don't increase $\gamma_{\mathrm{SB}}$ but potentially $\gamma_{\mathrm{CB}}$. Indeed, while the upper bound $\gamma_{\mathrm{SB}}$ decreases to 13.3 using the first 20 samples, $\gamma_{\mathrm{CB}}$ increases to 74.7 using the first 6 samples and LMI 17) even becomes infeasible for more samples. This observation is due to the high signal-to-noise-ratio of the measured trajectory for $t \geq 5$. Note that all optimization problems in this example are solved in less than a second on a Lenovo i5 notebook.

\section{B. Example 2}

The $\ell_{2}$-gain of the system

$$
\left[\begin{array}{l}
x_{1}(t+1) \\
x_{2}(t+1)
\end{array}\right]=\left[\begin{array}{l}
-0.5 x_{1}+0.3 x_{2}^{2}+0.2 x_{1} x_{2} \\
0.4 x_{2}+0.1 x_{2}^{2}-0.2 x_{1}^{3}+u
\end{array}\right](t)
$$

with $x_{1}^{2} \leq 1, x_{2}^{2} \leq 1$, and $u^{2} \leq 1$ is examined. Given the ground-truth system, we determine 2.1 as an upper bound of the $\ell_{2}$-gain by SOS optimization. The noise of the data (5) exhibits constant signal-to-noise-ratio $\left\|\tilde{d}_{i}\right\|_{2} \leq 0.02\left\|\tilde{x}_{i}\right\|_{2}$. Furthermore, $x(0)=\left[\begin{array}{ll}-1 & -1\end{array}\right]^{T}$ and we apply the input signal $u(t)=0.7 \sin \left(0.002 t^{2}+0.1 t\right)$ such that the system is excited over the whole time horizon. Moreover, we assume $z(x, u)=\left[\begin{array}{lllllll}x_{1} & x_{2} & x_{1}^{2} & x_{2}^{2} & x_{1} x_{2} & x_{1}^{3} & u\end{array}\right]^{T}$, i.e., the unidentified model (4) contains 14 unknown coefficients and more monomials than is required to describe the ground-truth system.

Using the first 30 noisy samples of the input-state trajectory, we calculate the bounds $\gamma_{\mathrm{SB}}=3.8$ and $\gamma_{\mathrm{CB}}=11.1$. With 300 data samples available, we can reduce the upper bound $\gamma_{\mathrm{SB}}$ to 2.3 and $\gamma_{\mathrm{CB}}$ to 3.6 . The advantage of Theorem 15 is that computation time to solve its optimization problem is about two seconds while solving the SOS optimization problem of Corollary 9 takes now about 10 minutes.

\section{CONCLUSIONS}

We established two set-membership frameworks to check whether a polynomial system is dissipative without an explicitly identified model but directly from noise-corrupted inputstate measurements. The first framework provides a dataefficient but computationally expensive condition for separately bounded noise using standard SOS optimization. The second framework considers cumulatively bounded noise to deduce a more computationally attractive LMI condition with SOS multipliers, which corresponds partially to a generalization of [5] for polynomial systems. Subject of future research, we extend the results to find optimal dissipativity properties as conic relations [7] or nonlinearity measures [18]. Furthermore, the extension of the presented frameworks for input-output measurements might be interesting.

\section{REFERENCES}

[1] H. K. Khalil. Nonlinear Systems. Prentice Hall, 2002.

[2] J. C. Willems. Dissipative dynamical systems part I: General theory. Arch. Rational Mech. Anal. 45, 321-351, 1972.

[3] A. Romer, J. Berberich, J. Köhler, and F. Allgöwer. One-shot verification of dissipativity properties from input-output data. IEEE Control Systems Letters, vol. 3, pp. 709-714, 2019.

[4] H. J. van Waarde, M. K. Camlibel, and M. Mesbahi. From noisy data to feedback controllers: non-conservative design via a matrix s-lemma. arXiv preprint arXiv:2006.00870 2020.

[5] A. Koch, J. Berberich, and F. Allgöwer. Provably robust verification of dissipativity properties from data. arXiv preprint arXiv:2006.05974. 2020.

[6] J. M. Montenbruck and F. Allgöwer. Some Problems Arising in Controller Design from Big Data via Input-Output Methods. In Proc. 55th IEEE Conf. on Decision and Control, pp. 6525-6530, 2016.

[7] G. Zames. On the Input-Output Stability of Time-Varying Nonlinear Feedback Systems. Part I: Conditions Derived Using Concepts of Loop Gain, Conicity, and Positivity. IEEE Trans. Automat. Control, 11(2):228238, 1966

[8] T. Martin and F. Allgöwer. Iterative data-driven inference of nonlinearity measures via successive graph approximation. Accepted for publication in Proc. 59th IEEE Conf. on Decision and Control, 2020. (arXiv preprint arXiv:2004.11746).

[9] M. Sharf. On the Sample Complexity of Data-Driven Inference of the $\mathcal{L}_{2}$-gain. arXiv preprint arXiv:2003.00238 2020.

[10] M. Guo, C. D. Persis, and P. Tesi. Learning control for polynomial systems using sum of squares. arXiv preprint arXiv:2004.00850 v $1,2020$.

[11] M. Milanese and C. Novara. Set Membership identification of nonlinear systems. Automatica, 40(6):957-975, 2004.

[12] A. Papachristodoulou and S. Prajna. A Tutorial on Sum of Squares Techniques for Systems Analysis. In Proc. American Control Conference, pp. 2686-2700, 2005.

[13] G. Chesi, A. Garulli, A. Tesi, and A. Vicino. Homogeneous Polynomial Forms for Robustness Analysis of Uncertain Systems. Springer, 2009.

[14] M. D. Choi, T. Y. Lam, and B. Reznick. Sums of squares of real polynomials. In Proc. Symp. in Pure Math., 58(2):103-126, 1995. 
[15] W. Tan. Nonlinear Control Analysis and Synthesis using Sum-of-Squares Programming. Ph.D. thesis, University of California, Berkeley, 2006.

[16] J. Löfberg. YALMIP: A Toolbox for Modeling and Optimization in MATLAB. In Proc. CACSD Conf., 2004.

[17] C. W. Scherer and S. Weiland. Linear matrix inequalities in control, Lecture Notes. Dutch Institute for Systems and Control, Delft, the Netherlands, 2000.

[18] T. Martin and F. Allgöwer. Nonlinearity measures for data-driven system analysis and control. In Proc. 58th IEEE Conf. on Decision and Control, pp. 3605-3610, 2019. 